\title{
Test and Experiment of Bio-Medical Image Restoration Using Enhanced Image DCT Fusion Techniques
}

\author{
Tushar ${ }^{1}$ and Abhijit Nayak ${ }^{2}$ \\ ${ }^{1} \mathrm{PhD}$ Scholar (Full-Time), USICT, GGSIPU, New Delhi, India \\ ${ }^{2}$ Dean, Bhagwan Parshuram Institute of Technology, Rohini, New Delhi, India
}

\begin{abstract}
The Image fusion is the process of integrating many images to generate a new image. In comparison to the source photos, the resulting image has greater information. The method of picture fusion has replaced clinical image combining as a relatively common practise these days. Medical imaging systems may be employed to provide complete sensory input, as well as diagnose and treat patients. To improve the accuracy and reduce the unpredictability of diagnosis, a bio medical images combines many pictures into a composite image. Image fusion is one of the most emerging research areas in recent time as the image has very significant role in various research work as well as in biomedical needs. There is various method which can have the capabilities to fuse the deformed image with certain level of accuracy. Centralized sensors have captured the images from two or more shots of one target. Further a refined image could be constructed through the image fusion and proposed DCT technique over the biomedical images. This research explored the DCT technique with auxiliary technique which fused the biomedical images through MATLAB GUI. The programming code has been build behind the GUI, which has been integrated with DCT technique.
\end{abstract}

KEY WORDS: IMAGE FUSION, MULTI-FOCAL IMAGE, DCT TECHNIQUES, BIO MEDICAL IMAGES.

\section{INTRODUCTION}

To expand on this definition, it, picture fusion involves combining uses several pictures to create a new image. more detail is presented in the output picture than is contained in the source images Clinical image combination has shifted to the technique of image fusion has, which is a very common practise these days. In order to supply all sensory input and to diagnose and treat, medical imaging systems may be used. Medical picture blends several photographs into a composite image to increase the accuracy and diminish the randomness of diagnosis. In addition to these two domains, image domains, image fusion takes place at a point in time. Spatial domain takes advantage of the fact that each pixel is made up of a multitude of its individual values and has additional values in the spectral domain. a method that

Biosc Biotech Res Comm P-ISSN: 0974-6455 E-ISSN: 2321-4007

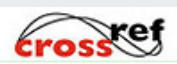

Identifiers and Pagination

Year: 2021 Vol: 14 No (5) Special Issue

Pages: 294-300

This is an open access article under Creative

Commons License Attribn 4.0 Intl (CC-BY). DOI: $h$ ttp://dx.doi.org/10.21786/bbrc/14.5/52 classifies sets of functions that deal with areas rather than the discrete points in space are F.T, DWT, DCT, Hadamard Transform, and the methods that deal with points in the spectral domain are DWT, and DCT (Delsy et al., 2020).

The picture of a certain scene may have missing data and two pictures of the same location may have opposite data. Although these two figures are shown as separate for the sake of simplicity, the processes and methods of fusion can allow for a better understanding. Thus, to picture fusion is used to improve spectral, spatial, or temporal, or also spectral or spatial resolution It is used in a number of fields, including remote sensing, satellite imagery, medical surveillance, field surveillance, and hidden weapons detection. Certain various techniques such as simple averaging, Stationary Wavelet Transform (SWT) (Aymaz \&t Köse, 2017), Discrete Wavelet Transform (DWT), Cosine Transform (DCT) (Babu \& Rao, 2017), etc. In good image fusion algorithm algorithms, each image's data should be retrieved from all contributors. It can minimise the number of new artefacts and reduce the noise.

Much of the time, the sensor images are inaccurate. The misalignment of the picture is caused by a variety of causes, including sensor geometries, sensor locations, and
Article Information

Received: $14^{\text {th }}$ Mar 2021 ccepted after revision: 30 $30^{\text {th }}$ May 2021 
sensor temporal capture rates that vary. It's possible that one source picture is a subset of another. It's possible that the same picture was captured by various cameras or sensors at different angles. Registration is the act of aligning source images in order to balance spatial correspondence between them, and it is a research subject in and of itself. Multiview, multifocal, multitemporal, and multimodal photos may all be used as source images for image fusion. Photos of the same scene taken at various periods are known as multi temporal images. In multimodal picture fusion, source images of various modalities are given. Multimodal images include CT and MRI pictures (Pichler et al. 2008), visible and infrared images, SAR and PAN images, and so on. Multi-focus image fusion combines photographs taken from the same view point but with separate focal settings to create a single image with all objects in focus.

One way to process the input images is to determine a source picture is to be expanded or another is to be completed domain expansion or frequency processing. Single-scale fusion approaches are used in the spatial context, while multi-scale fusion methods are used in the frequency domain. Picture processing in the spatial domain is performed with each and every pixel in mind. This pixel-based technique is used for image enhancement in filters including smoothening and sharpening (Daniel et al., 2017). Spectral transform methods are used to improve or modify images in the frequency domain. Using mathematical transformations such as DWT, DCT, DT-CWT, or NSCT, each source image is decomposed into a series of pictures. The fused coefficients are then calculated using a set of fusion laws. Finally, the inverse transform may be used to obtain the fused file. Fusion laws can be carefully framed in multiscale decomposition and reconstruction (Ma et al. 2018). If the fusion laws are ineffective, valuable knowledge from the source images will be lost, and new artefacts may be introduced. Image fusion may be categorised as feature level, judgement level, or pixel level fusion depending on the methodology used.

Feature level fusion applies the laws to regions instead of handling an image as a whole rather than using a set of dealing with it as a collection of pixels. Pixels are viewed as objects of their own right or as well as being areas of interest (ROI). The photographs are divided into various segments to yield a set of areas. Segmentation produces a finite closed interconnected series of segments. An alternative to the simple neighbourhood algorithm is the watershed. Segmentation is used after the operations to obtain region properties such as coloured areas, bounded areas, branches, margins, corners, patterns, and patterns. If the sub-target segmented spatial frequencies and target characteristics have been computed, the combined region's features are applied to the segmentation process is carried out.

Because several regions are sampled, the fused image would be a picture that has been given preference to the highest probability of including them, according to their arrangement around the original source pictures.
Centralized sensors are programmed to render decisions and can or noncentralized ones are operated, but all methodologies operate according to their own data and determine decisions are redirected to a central fusion module. All is done centrally, but no decisions get out of the core seats. As opposed to this, in a centralised sensor system, in decentralised systems, each node makes decisions based on the local data it collects from nearby nodes. Fuzzy logic is a classifier, various kinds of voting classifiers are also have been applied, and Bayesian processes, and so on and so forth (Velmurugan et al. 2018).

II. Review of Literature: Sreeja \& Saraniya (2021), present a multi-focal image in the cosine transform (expanded cosine images) which recently become highly popular for the increasing requirements on domain homogenization because of low complexity. is in fact, the method of fusion by DCT is most valuable in visual sensor networks (or where images have to be encoded), where the data is most likely to be troublesome. Spatial approaches have been used in a number of research projects to examine the issues in DCT (a 2D image data representation domain). It has been stated in the upcoming work that the energy of correlation in the DCT domain will be used as the criterion for fusion. The findings of the suggested technique are scrutinised by other techniques to see whether they can indeed mitigate those types of block selection errors and whether they can be verified using quantitative measurements.

Majhi \& Pal (2021), introduced a scheme for image retrieval is proposed which takes advantage of blocklevel geometric features is described in this paper. The block level features are removed after DCT and second level features are obtained in the first level element, picture block part computation, each block's magnitude is converted to an AC coefficient that allows for further significance in making an image come through. typically, in the design, the AC components are assumed to have the second level of features and then the DC features are implemented to obtain the higher ones After the first stage of matrix expansion, singular vectors and left singular vectors are generated from the AC components. SVD is then performed on the expanded components to create the second level.

Because the value on the matrix on the left contains just a single data point, certain mathematical parameters are calculated for the second stage of the scheme on determine values to the corresponding columns to the right. to all first and second stage functionality Wherever it may be, more importance is attached to the first (high level) aspect, wherever it may be, more emphasis is provided to the lesser feature, i.e. Also, all three-dimensional attribute extraction is performed separately gives the described in this model for three sets, providing more distinguished categories for the final scheme. using the five established techniques is done to calculate similarity of the various distances for their retrieval; after which, the findings are evaluated to determine the effectiveness of the proposed retrieval 
strategy on the contrary, retrieval work in the picture domain performs well due to the increasing by 50\% with increased experimental difficulties, the findings are consistent with prior results.

Yadav \& Amhia (2021), introduced Several techniques for lossless image fusion are investigated and described in this dissertation, most notably Image Threshold Expansion, De-combination, Comb-Warp, Comb-Warp Threshold, and Comb-Warping Thresholding. One of the primary purposes of this work is lossless image fusion algorithms that use tree structure background simulation as a key concept. To be more specific, they have to use distinct Cosine transform coefficients, their position in the picture, a variation of that sense, as well as a strength of samples, and their degree of contribution to the image, makes a difference, respectively. The study in this thesis involves analysis using DDCT background model, estimation, and assignment for DDT tree structure, as well as well as fusion and image retention for image reconstruction, the biggest benefit is efficiency, but other benefits include new types of non-destructive analysis, which will improve background modelling, and improving quality techniques that have better scalers for various gray-scale images: scientific, remote natural pictures. There has been a major growth in transmission of images in computer and smartphone. This study is explored image fusion research through DDCT very significantly.

Vakaimalar \& Mala (2019), introduced a multi-focus pictures that show more aspects of the same scene taken from various angles in two or more cameras. As a result, this approach is about producing a goodquality picture, the authorship candidate, discrete cosine transform and spatial frequency expansion are combined to find the individual with greater clarity. The algorithm suggested has no limitations as far as the number of photos is concerned. The second stage computes the average and maximum values from all the photos and processes the values only to the ones that are between these values. Then the two input images are first expanded by the Discrete Cosine Transform (DCT) followed by inverse Discrete Fourier Transform (IDFT). DCT coefficients are expanded to their greatest/ smallest extent and image fusion is accomplished using spatial frequency. Expanding the second stage of the algorithm, such as in the design of the Stationary Wavelet Transform, the Principal Component Analysis, and spatial fusion is useful in certain prior algorithms, such as regularisation. A new type of analysis proves that the new algorithm outperforms other methods in terms of image compression, utilising standard measurements as well as DCT and the most recent and efficient DCT techniques.

Vanitha et al. (2019), introduced a modern approach focused on fourth-order partial differential equations and discrete sine transform in stationary wavelet transform space (SWT) is explored for FPDE and MRI multimodal imaging. The first step is to expand the source images into base and information images by using a fourth- order differential equation. A final picture is obtained by taking the average of information photos based on their principal components. Next, the base images are used as the starting points for SWT decomposition. In this case, the four sub-band coefficients are computed using the Discrete Cosine Transform (DCT). The subsampling expansion phase is employed to acquire large sub-band coefficients. The final output is obtained by applying the inverse Discrete Cosine Transform (IDCT) followed by the inverse Squared Error Transform (SWT). The proposed methods for realising the fusion are compared with the actual ones to determine their similarities through analysing the output outcomes, it seems that the proposed approach does well in terms of quantitative metrics like mean, standard deviation, such as F-Measure, M-Measure, and T-measure.

Rao et al. (2019), accomplished an article over the photos which are associated; computed tomography (CT) scan, along with a magnetic resonance imaging (MRI) provide a clearer and more informative representation of the existing picture. In this article, numerous wavelet-based fusion methods are used, such as the discrete wavelet transform (DWT) and two others that use a different approach to the discrete cosine transform (DCT) as verification of accuracy of evidence; and CV-based and the stationary wavelet fusion (SW) include DWT in an incidental fashion and CV variation in image processing methods include, but SW verification to DCT consistency are used in CV-based and stationary wavelet fusion image processing, a few SWWT. Pleomorphic measurement metrics are used to distinguish unfused results that can be had from the separate ones that are imparted by these techniques of fusion Fused out of DWT accompanied by SWT applied to DCT image processing results in this DCT var is what performs the SWT in an expanded technique When potentiality DCT features are applied to an output, there is value-added data available by models created from models using fused techniques that help with the delivery of outcomes. Applying DCT, the concert layer bases are quick and less DCT in realitygrounded likeness computations. A detailed procedure for DCT-based approach is available to discover different and expanded views of the same thing is illustrated and analysed in this attempt, as DCT fused results outweigh the other fusion methodologies.

Paramanandham \&t Rajendiran (2018), introduced a novel fusion method for combining infrared and visible imaging techniques is introduced in this publication. In other words, the audience would still have to make the additional effort to piece the sensor outputs together. A technique for imaging two or more channels in a single picture, known as discrete cosine transform (DCT) is suggested for this paper to allow for security applications which integrates the infrared and visible channels to generate a single insightful merged image (WBIFI). The weighting method in particle-swarm length optimization is applied to particles (small pieces of data) is done with particle swarm optimization (PSO). These special weighting constants are used to combine visible and infrared DCT coefficients to fabricate new images for 
printing. The inverse DCT technique is used to produce the fused or expanded images. An adaptive histogram equalisation process is used to get a more comprehensive interpretation of the picture while at the same time as it best distinguishes the objective. Although various quantitative measures are used in this context, the relative statistics such as the standard deviation, spatial frequency, entropy, and gradient mean are assessed. The findings demonstrated the algorithm's superiority over existing well-known approaches such as expandable writing and skip- wave learning, published in existing literature.

Mahapatra et al. (2018), introduced an Image Fusion which has no movement, and complicates the work of the wireless sensor nodes since they have to wait to get their sources. If there are photographs available at the source, they may be of low quality, or they may include redundancies. Using picture fusion techniques, a clearer and smaller image may be produced that depicts the origins of the unclear and diffused image. The method discussed in this paper uses domain variance as a key variable to take into account more fully different regions while seeking to expand attention into a multifocal picture. Due to the versatility of our approach, this methodology, their product can be used in realtime applications. To the experiment's already shown outcome. They have now added further evidence. The latest validation of their approach has obtained, as of its accuracy and sophistication. Amin-Naji \& Aghagolzadeh (2018), introduced relevant knowledge from the inputs and pulling it together to work on a common target are two separate, but complementary approaches to a goal. With varying depth of field, multiple camera focal depths may be used to get distinct photographs.

Many strategies that use a multi-focal picture fusion with various focal points have recently been applied, because of the discovery of how to quantify the focal points in the spatial domain. System-sensor systems because DCT imagery can be effectively be displayed as separate sharpened with no user control over the gain. As far as studies on DCT domains are concerned, most scholars are concerned, the most are focusing on calculations for defocus and domain-expansion. This entailed substituting the spatial domain processes with DCT processes, hence, a lot of scientists used new and developing methods which performed spatial domain expansion. This may lead to limitations when working in DCT domains, since of previous studies only employ such appropriate blocks of the optical signal sources based on their focus measuring criteria. as opposed to prior work which recommended only for specific energies. As it has been shown that these suggested approaches do not need appropriate block selection, these disadvantages are important. By using the approaches, they recommend, to see outcomes in a fair to extremely favourable as compared to the results of the ones previously used.

Kulkarni \&t Bichkar (2018), attempted to combine details from separate pictures of a scene to extract valuable information. Many different data-processing methods, such as the Discrete Cosine Transform (DCT), Kritèktela Transform (K-T) and the Walsh Transform (WT), have been used in image fusing operations. Using these transformations, this paper can help to analyse the consistency of the result. used previously to evaluate image quality with indices that involve, respectively, mean, variance, RMSSIM, mean squared error PSNR, and MSE, but they can also look at one additional measure: peak signal-to-to-noise ratio (PSNR). This article takes an opportunity to compare these fusion methods in order to provide a definitive understanding of their strengths and weaknesses. Additional analysis of some standard image files indicates that DWT and DCT performs well in comparison to the other standard methods.

Amin-Naji et al. (2017), introduced an optimal way of merging of images in the Discrete Cosine Transform (DCT) can be achieved by using low-complexity expansion, which uses a small amount of time and resources. In digital sensor networks, it is particularly difficult to expand and reduce many image files as they are stored in JPEG format (VSN). This paper reports a lower complexity image quality than usual image expansion for wide-field image fusion using DCT in which the performance image quality is increased. A current implementation of our proposed approach is ideal for real-time implementations since it's made in the DCT domain. It is very stable, as long as there is nothing to distract it.

This approach is based on the Singular Value Decomposition (SVD) of $8 \times 8$ space, which decomposes 8 block magnitudes into their singular values. To obtain the geometric mean (a mean that is a multiple of the most singular values out of 8), they use an expanded criterion for singular value identification. the block that most strongly resembles the target block of other than the rest of the blocks is chosen to serve as the centred This is when these blocks are employed in the construction of the final picture. This algorithm works in both the DCT (discrete cosine transform) and PTS (progressive transmission scan) domains. A number of studies and trials comparing the proposed approaches and the results with those of the prior methods in noise-filled and quiet settings have been performed, and the results have all shown an improvement in picture quality and stability.

Babu \&t Rao (2017), attempted to fuse several images for the single picture representation (measured using different instruments such as MS and Panchromatic and Panchromatic), and the goal is to communicate greater detail and variety about the signal intensity and the various signal contributions of different sensors. $T$ verification using two DWT extended versions, varia). In this article, discrete cosine transforms (DCT) and specialised DWT techniques are analysed and checked against the implementation of stationary wavelet transform variants (known as $\mathrm{T}$ variance checks), referred to as ST called a (which is also a variation of) dubbed stationary transform. Actualization of these findings, achieved by a combination of expansion and assessment methods, are inspected using conventional measurements. In general, the practical implementations, 
DCT proper discrete and image expansions may be done using the grounded-expansion rules of the general DCT, whereas the resulting process images are more detailed and performant. This investigation, by way of the semantic Web user vocabulary toolkit, elucidates a practise of radiating coloured system-integrated images and shows that best SWT fused effects are superior to those of other fusion methods.

Kaur et al. (2015), presented a valuable knowledge from more than one perspective on image fusion. Use PCA to detect components from motion images to see performance benefits and gains with a measure of rapidness of both speed and effectiveness in real-time for such devices. a multi-dimensional representation (PCA, emphasis) is suggested that has a great capacity to represent the variation in a picture in an approach which can be fused together using domain-specific multi-focus images A new approach to alleviate the AC-specific problems encountered due to the discrete cosine transform is presented in this article, one that incorporates alternate current normalisation techniques (which is known to be inherently strong in the Cosine domain) into the discrete cosine transform (DCT) domain to minimise artefacts that result from the latter. Since sharpness could be reduced as a side effect of the fusion, improved results were obtained by incorporating an enhancement algorithm that takes care of the issue.

\section{Image Fusion with DCT (Discrete Cosine Transform)} Techniques: There are various image fusion techniques like DCT (discrete cosine transform) (Ravi \&t Krishnan, 2018), discrete wavelet transforms (DWT) (Jinju e al. 2019), Walsh Transform (WT) (Kekre et al. 2012) and Discrete Fourier Transform (IDFT). These techniques already used to find the image enhancement through Image Fusion techniques. Existing techniques has no integrated platform to provide a generation of quality Images from hazed images. This research needs a use friendly interface which has to perform the Image fusion with more techniques in single interface system.

\section{METHODOLOGY}

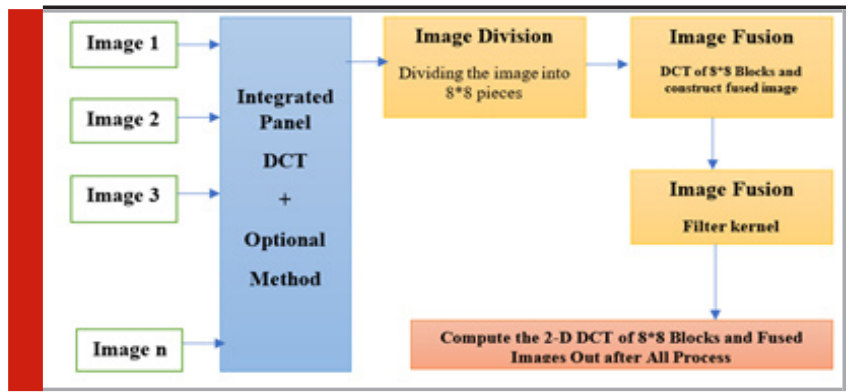

As the above methodology illustrate that the fusion process with DCT (Discrete Cosine Transform) with subsidiary enhanced support fusion techniques (Optional Method). The image has been divided as $8 * 8$ matrix pieces. Then The filtering process of the fused method has been applying to get the best pixel level of the changed information. Finally compute the fused images and display the merged images as final best extracted
Fused image with the optional method.

\section{Multi-focus Image Algorithm}

Start function (multi-focus);

\{

Select Img 1 \&t Img 2;

Pixlet Img 1 \&t img 2;

Covert_Low_Info rgb2gray (Img1 \& img 2);

Display ImgSize (Img1 \& Img 2);

Level-Shift: DCT;

Div (Img1 \& Img 2)/8*8;

Fused_Img $=$ Fused DCT+Variance+SVD+CORR (Img 1

\& Img 2);

Image_Out= InverseDCT (FusedImg);

Show (Image_Out);

\}

Exit Function (multi-focus);

V. Result and Simulation: The GUI has been designed in MATLAB and all formulation has been coded behind the interface. The above figure is the basic layout designed to perform the image fusion using GUI designed MATLAB Layout for user end. This has two inputs of image suing the selected button as one has left and right side. As the above simulation illustrate the fusion image at the end of process achieved which has best pixels of the selected image 1 and image 2. The GUI has various method of DCT with VARIANCE, VARIANCE+CV, CORR, CORR+CV, SVD, SVD+CV, SVD+CV, CORR_ENG and CORR_ENG+CV. Each method has been successfully tested and perform on the images (Data based Taken from GitHub).

Figure 1: The GUI constructed for Image Fusion

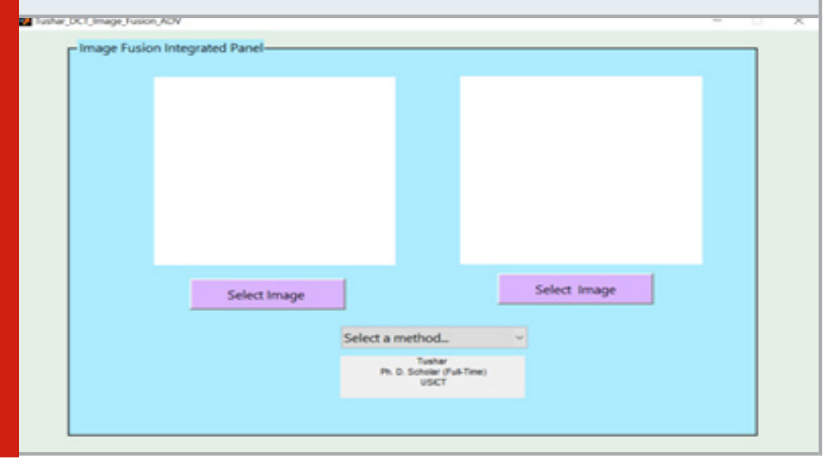

Figure 2: Image 1 and Image 2 (Both of same in different angle hazed image)

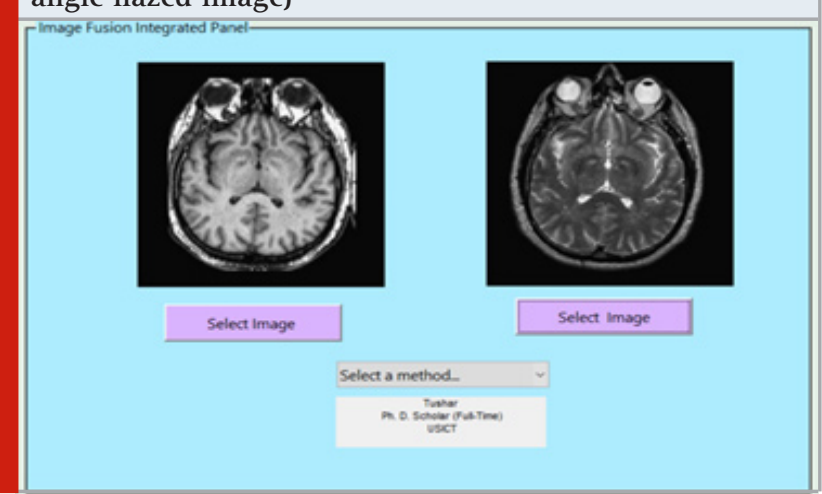


VI. Significance of the Research: As the bio medical images feature visibility is offered as a method to assess the quality of a picture. This strategy is restricted by the fact that the human visual system is impacted by the contrast. Therefore, the visibility of image may be more closely approach to general considerably greater visibility. It is offered a medical picture fusion strategy based on the DCT transform. The suggested strategy was shown to be effective based on the results of the experiments. In the fusing of data, it beats traditional fusion approaches. photographs of various medical conditions the suggested image fusion method is as follows:

Figure 3: Fused image out after the apply the DCT+ Var+ CV.

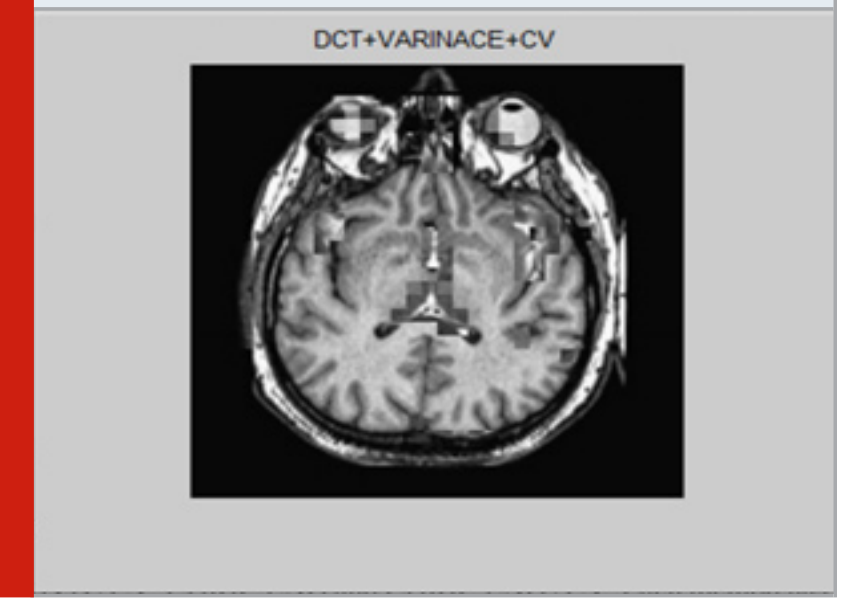

a. Proposed method is effective, efficient, and practical.

b. The suggested strategy is capable of preserving the crucial structure.

c. Medical diagnostics applications are very applicable due to various method in the User GUI.

\section{CONCLUSION}

The Image Fusion integrates a lot of pictures and produces a new picture. The final picture contains more information than the original photographs. The image fusion approach has replaced the clinical picture which nowadays combines as a rather regular practise. Medical imaging systems may be used both for the whole sensory input and for the diagnosis and treatment of patients. A bio medical image combines multiple photos with a composite image to increase accuracy and lessen the unpredictability of the diagnosis. Fusion of images is one of the rising fields of study in recent years since the picture plays a very important function both in different research and medicinal demands. The visibility of a biomedical pictures is presented as a way of assessing the quality of an image. Because the human visual system is influenced by contrast, this method is limited. As a result, the image's visibility might be closer to that of the general population. A medical image fusion technique based on the DCT transform is available. There are many methods that can fuse the distorted picture with specific precision. Two or more shots of a same target have been captured by centralised sensors. Through image fusion and a suggested DCT approach of biological images provide improved images through MATLAB GUI. The DCT technology and its subsidiary technique on biomedical images were fused successfully in this research.

\section{REFERENCES}

Amin-Naji, M., \&t Aghagolzadeh, A. (2018). Multi-focus image fusion in DCT domain using variance and energy of Laplacian and correlation coefficient for visual sensor networks. Journal of AI and Data Mining, 6(2), 233250.

Amin-Naji, M., Ranjbar-Noiey, P., \&t Aghagolzadeh, A. (2017, November). Multi-focus image fusion using singular value decomposition in DCT domain. In 2017 10th Iranian Conference on Machine Vision and Image Processing (MVIP) (pp. 45-51). IEEE.

Aymaz, S., \&t Köse, C. (2017, November). Multi-focus image fusion using stationary wavelet transform (swt) with principal component analysis (pca). In 2017 10th International Conference on Electrical and Electronics Engineering (ELECO) (pp. 1176-1180). IEEE.

Babu, C. R., \&t Rao, D. S. (2017). Comparison of discrete wavelet transform (DWT), discrete cosine transform (DCT) and stationary wavelet transform (SWT) based satellite image fusion techniques. Int J Cur Res Rev, 9, 49-53.

Babu, C. R., \&t Rao, D. S. (2017). Comparison of discrete wavelet transform (DWT), discrete cosine transform (DCT) and stationary wavelet transform (SWT) based satellite image fusion techniques. Int J Cur Res Rev, 9, 49-53.

Daniel, E., Anitha, J., Kamaleshwaran, K. K., \&t Rani, I. (2017). Optimum spectrum mask based medical image fusion using Gray Wolf Optimization. Biomedical Signal Processing and Control, 34, 36-43.

Delsy, T. T. M., Nandhitha, N. M., \&t Rani, B. S. (2020). Feasibility of spectral domain techniques for the classification of non-stationary signals. Journal of Ambient Intelligence and Humanized Computing, 1-8.

Jinju, J., Santhi, N., Ramar, K., \&t Bama, B. S. (2019). Spatial frequency discrete wavelet transforms image fusion technique for remote sensing applications. Engineering Science and Technology, an International Journal, 22(3), 715-726.

Kaur, P., Singh, M., \& Singh, B. Evaluating the performance of integrated DCT and PCA based image fusion for multi-focus images.

Kekre, H. B., Sarode, T., \&t Dhannawat, R. (2012). Implementation and comparison of different transform techniques using Kekre's wavelet transform for image fusion. International Journal of Computer Applications, 44(10), 41-48.

Kulkarni, J. S., \& Bichkar, R. S. (2018). Comparative Analysis of Image Fusion Using DCT, DST, DWT, Walsh Transform and Kekre's Wavelet Transform. In 
International Conference on Intelligent Computing and Applications (pp. 227-235). Springer, Singapore.

Ma, T., Ma, J., Fang, B., Hu, F., Quan, S., \& Du, H. (2018). Multi-scale decomposition-based fusion of infrared and visible image via total variation and saliency analysis. Infrared Physics \& Technology, 92, 154-162.

Mahapatra, S., Sa, K. D., \&t Dash, D. (2018, April). DCT Based Multi-focus image fusion for wireless sensor networks. In 2018 Second International Conference on Inventive Communication and Computational Technologies (ICICCT) (pp. 871-875). IEEE.

Majhi, M., \& Pal, A. K. (2021). An image retrieval scheme based on block level hybrid DCT-SVD fused features. Multimedia Tools and Applications, 80(5), 7271-7312. Paramanandham, N., \&t Rajendiran, K. (2018). Infrared and visible image fusion using discrete cosine transform and swarm intelligence for surveillance applications. Infrared Physics \&t Technology, 88, 13-22.

Pichler, B. J., Judenhofer, M. S., \&t Pfannenberg, C. (2008). Multimodal imaging approaches: Pet/ct and pet/ mri. Molecular Imaging, I, 109-132.

Rao, D. S., Babu, C. R., Kumar, Y. N., Rajasekhar, N., \&t Ravi, T. Medical Image Fusion Using Transform Based Fusion Techniques. International Journal of Recent Technology and Engineering, 8(2).
Ravi, P., \& Krishnan, J. (2018). Image enhancement with medical image fusion using multiresolution discrete cosine transform. Materials today: proceedings, 5(1), 1936-1942.

Sreeja, G., \& Saraniya, O. (2021). Energy Efficient MultiFocus Image Fusion in DCT Domain. In Advances in Smart System Technologies (pp. 713-718). Springer, Singapore.

Vakaimalar, E., \&t Mala, K. (2019). Multi-focus image fusion scheme based on discrete cosine transform and spatial frequency. Multimedia Tools and Applications, 78(13), 17573-17587.

Vanitha, K., Satyanarayana, D., \& Prasad, M. G. (2019, July). A new hybrid medical image fusion method based on fourth-order partial differential equations decomposition and DCT in SWT domain. In 2019 10th International Conference on Computing, Communication and Networking Technologies (ICCCNT) (pp. 1-6). IEEE.

Velmurugan, S. P., Sivakumar, P., \&t Rajasekaran, M. P. (2018). Multimodality image fusion using centre-based genetic algorithm and fuzzy logic. International Journal of Biomedical Engineering and Technology, 28(4), 322348.

Yadav, P. S. P., \&t Amhia, H. (2021). A Review Article of Image Fusion Technique Using Wavelet Transform. 